\title{
Location and Routing Models for Emergency Response Plans with Priorities
}

\author{
Ali Oran ${ }^{1}$, Kiat Chuan Tan², Boon Hooi $\mathrm{Ooi}^{2}$, Melvyn Sim ${ }^{3}$, and Patrick \\ Jaillet $^{4}$ \\ 1 SMART, Singapore ${ }^{\star \star}$ \\ aoran@smart.mit.edu, \\ 2 DSO National Laboratories, Singapore \\ \{tkiatchu, oboonhoo\}@dso.org.sg \\ 3 NUS Business School, Singapore \\ melvynsim@nus.edu.sg \\ 4 EECS \& ORC MIT, Cambridge MA, USA \\ jaillet@mit.edu
}

\begin{abstract}
In emergency planning, consideration of emergency priorities is a necessity. This paper presents new formulations of the facility location problem (FLP) and vehicle routing problem with time windows (VRPTW) with considerations of priority. Our models ensure that higher priority locations are considered before the lower priority ones, for both facility and routing decisions. The FLP is solved using an MIP solver, while a tabu search based metaheuristic is developed for the solution of the VRPTW. Under a set of possible emergency scenarios with limited emergency resources, our models were able to serve higher priority locations better than the much utilized Maximal Coverage Location Problem (MCLP) model. We also present preliminary work and results for an integrated location-routing analysis which improves service results further.
\end{abstract}

Keywords: Emergency Planning, Facility Siting, MCLP, VRPTW

\section{Introduction}

Increasing population growth and high level of urbanization have increased the impacts of disasters, whether natural or man-made. Consequently, there is an increasing need for new methods to better prepare for and manage emergencies. In general, a good emergency response plan (ERP) must yield an efficient and immediate emergency response to locations that will be affected by the actual disasters. As a first stage of an ERP, in the preparation stage, emergency facilities must be sited at optimal locations, and the emergency vehicles (eg. Fire Trucks, First Aid Vehicles etc), that will be bringing relief material to emergency points from these facilities, be allocated to the right facilities. Later, during the actual emergencies, within short decision times, optimal routes for emergency vehicles must be found, and updated dynamically when new information about

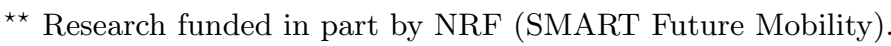


the emergencies becomes available. Both problems have been studied in detail in operations research, under the broader problems of FLPs, and VRPs (see surveys [1], [2]), respectively.

When planning for an ERP, all possible hazards must be considered as part of a thorough risk assessment and prioritized on the basis of impact and likelihood of occurrence. Otherwise, treating all hazards the same in terms of planning resource allocation would ultimately lead to failure [3]. Priority considerations are particularly essential when planning for emergencies with limited resources. In such situations trade off decisions must be made about which emergencies to receive the available resources based on the priority of emergencies. For instance, for fire-related emergencies, it would be prudent to consider serving a petrochemical plant with fire trucks before less fire-sensitive areas because of the possible risks associated with such an emergency in that plant. While essential in emergency planning, priority related problems have not been studied in detail in FLPs except a few studies, e.g. [4].

A major challenge for developing ERPs is the uncertainties involved with emergencies, such as the time and location of their occurrence. In order to account for the uncertainties the 'backup coverage' concept of Hogan and ReVelle [5] could be utilized. It constructs a more reliable or robust facility network, such that in the event of facility failures (or busyness) any remaining facilities can continue to provide a sufficient level of coverage [6]. An ERP with priority considerations can particularly benefit from backup coverage utilization if locations with high risks (high priority) could be covered by more than one facility.

It has been noted that for problems where different customers will be served simultaneously with vehicles from the sited facilities, such as the ERP problem mentioned above, the location and routing decision will be strongly interrelated [7]. This dependency suggests that FLP results might not be indeed optimal when considered without the possible service effectiveness of vehicles dispatched from the sited facilities. For instance, the optimality of an emergency facility location depends on how effectively the vehicles can respond to emergency calls. In general, a combined FLP-VRP analysis could give the planner a more accurate measure of the location decisions for problems that involve the delivery of goods with vehicles dispatched from the facilities. For an ERP, such a combined assessment could improve the reliability of the proposed emergency facility plans.

In this paper we introduce a new methodology for the development of ERPs. Overall, our approach consists of a facility location model, MCLP-PB, built on the MCLP with priority considerations and backup coverage, and a vehicle routing model, VRPTW-P, built on the VRTPTW with priority considerations. VRPTW-P is also used to analyze MCLP-PB results, under different demands characterized by possible emergency scenarios. For the solution of VRPTW-P, we present a fast and accurate metaheuristic that could update vehicle routes dynamically, and which could be utilized in real life emergencies where computation time is critical. Finally, as an extension of our approach, we present an integrated location-routing approach, which finds location and routing decisions in a feedback loop fashion rather than a sequential analysis. 


\section{Problem Formulation and Model Development}

Consider a network $G(V, A)$, with $V$ being the set of locations of interest, and $A$ the set of arcs in the network representing roads in the physical space. In this network, we are given a set of potential sites for emergency facilities $\mathcal{J} \subset V$, and the set of emergency sites (points) $\mathcal{I} \subset V$. To develop a general method, we consider multiple types of emergencies, with each type demanding service by a particular type of emergency vehicle, e.g. fire emergencies demanding fire trucks. The type of an emergency vehicle is represented by an element of the index set $\mathcal{K} \doteq\{1,2, \ldots\}$. An emergency point $i \in \mathcal{I}$ is said to be covered by site $j \in \mathcal{J}$ if and only if the shortest distance (or travel time) from $j$ to $i$ is less than a preset coverage standard [1]. We define, $N_{i}^{k} \doteq\{j \in$ $\mathcal{J} \mid j$ can cover $i \in \mathcal{I}$ by vehicle type-k, $k \in \mathcal{K}$ under distance (time) standards $\}$. Other parameters and decision variables are defined below.

\section{Parameters:}

$Q$ : number of available facilities for operation,

$P^{k}$ : number of available type-k vehicles for allocation to facilities,

$p_{i}^{k}$ : priority (value) of emergency point $i \in \mathcal{I}$ for vehicle type-k,

$d_{i}^{k}$ : number of distinct facilities required to cover $i \in \mathcal{I}$ with type- $k$ vehicles,

$\alpha_{i}^{k}$ : weight for type-k emergency at $i \in \mathcal{I}$.

\section{Decision Variables:}

$y_{j} \doteq \begin{cases}1 & \text { if a facility is sited at } j \in \mathcal{J} \\ 0 & \text { otherwise }\end{cases}$

$x_{j}^{k} \doteq \begin{cases}1 & \text { if a type- } k \text { vehicle, } k \in \mathcal{K}, \text { is allocated to the facility at } j \in \mathcal{J}, \\ 0 & \text { otherwise. }\end{cases}$

$w_{i}^{k} \doteq \begin{cases}1 & \text { if } i \in \mathcal{I} \text { is covered by a type-k emergency vehicle, } k \in \mathcal{K}, \\ 0 & \text { otherwise. }\end{cases}$

Below, we first reintroduce the basic MCLP model with the notion of priority, which we call MCLP-P, in order to consider it as a benchmark against our model.

$$
\begin{array}{ll}
\max _{y_{j}, x_{j}^{k}, w_{i}^{k}} \sum_{k \in \mathcal{K}} \sum_{i \in \mathcal{I}} \alpha_{i}^{k} w_{i}^{k} & \\
\text { s.t }: & \sum_{j \in J} y_{j} \leq Q, \\
& \sum_{j \in \mathcal{J}} x_{j}^{k} \leq P^{k}, \quad \forall k \in \mathcal{K}, \\
& x_{j}^{k} \leq y_{j}, \\
& \sum_{j \in N_{i}^{k}} x_{j}^{k} \geq w_{i}^{k}, \quad \forall k \in \mathcal{K}, \forall i \in \mathcal{I} .
\end{array}
$$


(2) and (3) are the constraints for the available number of facilities, and vehicles, respectively. (4) avoids allocating a vehicle at a point with no facility, (5) ensures that a point $i \in \mathcal{I}$ is covered by a type-k vehicle, only if there is at least one type$\mathrm{k}$ vehicle already sited at a facility that can cover $i$ under the distance (time) standard. The difference between MCLP-P and MCLP comes from the definition of weight $\alpha_{i}^{k}$ in (1). In MCLP-P, we define $\alpha_{i}^{k}$ by considering the priority of type-k emergency at location $i \in \mathcal{I}, p_{i}^{k}$. One particular case is the formulation for absolute priority, where $\alpha_{i}^{k}$ should be defined such that the coverage of an emergency point with priority value $p$ is always worth more than the coverage of all points with priorities less than $p$. In such analysis, the MCLP-P model would yield a solution with as many high priority points covered as possible.

\subsection{Location Model, MCLP-PB}

As mentioned earlier, our location model is based on introducing extra coverage for emergencies of high priority. We assume that the number of distinct coverage demands, $d_{i}^{k}$, is given for each emergency point $i \in \mathcal{I}$ and emergency type $k \in \mathcal{K}$. To introduce the extra coverage considerations we redefine the objective function as a penalty function as shown (6). The difference term inside the square brackets is the unsatisfied distinct coverage demand of a type-k emergency at location $i \in \mathcal{I}$. The weight $\alpha_{i}^{k}$ is again used to reflect the priority of type-k emergency at point $i$. If the absolute priority case is considered, then minimizing the objective function ensures that higher priority points, together with their backup coverage requirements, are covered first. (7),(8) and (9) are also the first three constraints of the MCLP-P model, and are introduced for the same requirements. The formulation below is transformed into a linear form by introducing the difference term in brackets as a new variable, and adding extra constraints accordingly.

$$
\begin{aligned}
& \min _{y_{j}, x_{j}^{k}} \sum_{k \in \mathcal{K}} \sum_{i \in \mathcal{I}} \alpha_{i}^{k}\left[d_{i}^{k}-\sum_{j \in N_{i}^{k}} x_{j}^{k}\right]^{+}, \text {where }[f(.)]^{+} \doteq \begin{cases}f(.) & \text { if } f(.) \geq 0, \\
0 & \text { otherwise. }\end{cases} \\
& \text { s.t }: \sum_{j \in J} y_{j} \leq Q \\
& \quad \sum_{j \in \mathcal{J}} x_{j}^{k} \leq P^{k}, \quad \forall k \in \mathcal{K}, \\
& x_{j}^{k} \leq y_{j}, \quad \forall k \in \mathcal{K}, \forall j \in \mathcal{J} .
\end{aligned}
$$

\subsection{Routing Model, VRPTW-P}

Our routing model is an extension of the VRPTW formulations of Desrosiers et al. [8] with the notion of priority, and the relaxation of the constraint for serving every demand. We assume routing analysis succeeds facility solutions, so that $\mathcal{J}^{k} \subset \mathcal{J}$, the set of facilities that house type-k vehicles, is already found for all $k \in \mathcal{K}$. New terms consistent with the ones defined earlier are introduced below. 
Sets:

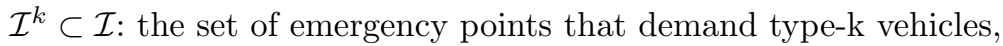
$\mathcal{A}^{j, k} \doteq\left\{(m, n) \in\left(\mathcal{I}^{k} \cup\{j\}\right) \otimes\left(\mathcal{I}^{k} \cup\{j\}\right) \mid \delta_{m j} \leq C, \delta_{n j} \leq C\right\}, j \in \mathcal{J}^{k}, k \in \mathcal{K}$.

\section{Parameters:}

$\beta_{i}^{k}$ : weight for serving type-k emergency at $i \in \mathcal{I}^{k}$,

$\Delta_{i}^{k}$ : amount of relief material demanded from a type-k vehicle at $i \in \mathcal{I}^{k}$,

$D^{k}$ : range (distance) capacity of type-k vehicles,

$C^{k}$ : emergency relief material capacity of type-k vehicles,

$\delta_{m n}$ : minimum distance between $m \in(\mathcal{I} \cup \mathcal{J})$ and $n \in(\mathcal{I} \cup \mathcal{J})$,

$\left[e_{i}, l_{i}\right]$ : the time window at $i \in \mathcal{I}$ within which the emergency must be responded, $t_{m n}^{k}$ : travel time for a type-k vehicle on $\operatorname{arc}(m, n), m \in(\mathcal{I} \cup \mathcal{J})$ and $n \in(\mathcal{I} \cup \mathcal{J})$, $s_{i}^{k}$ : service time for a type-k vehicle at $i \in \mathcal{I}$, and $s_{i}^{k}=0, \forall i \in \mathcal{J}$, $\bar{t}_{i j}^{k} \doteq t_{i j}^{k}+s_{i}^{k}$.

\section{Decision Variables:}

$z_{m n}^{j k} \doteq \begin{cases}1 & \text { if the type-k vehicle housed at } j \in \mathcal{J}^{k} \text { travels on } \operatorname{arc}(m, n) \in A^{j, k} \\ 0 & \text { otherwise. }\end{cases}$ $T_{n}^{k} \doteq\left\{\begin{array}{l}\text { Service start time at } n \text { if } n \in \mathcal{I}^{k}, \text { for type-k vehicle } \\ \text { Return time to facility } n \text { if } n \in \mathcal{J}^{k}, \text { for type-k vehicle }\end{array}\right.$

We formulate VRPTW-P as:

$$
\begin{array}{lc}
\max _{z_{m n}^{j k}} \sum_{k \in \mathcal{K}} \sum_{j \in \mathcal{J}^{k}} \sum_{(m, n) \in \mathcal{A}^{j, k}} \beta_{n}^{k} z_{m n}^{j k} & \quad \forall k \in \mathcal{K}, \forall m \in \mathcal{I}^{k}, \\
s . t: \sum_{j \in \mathcal{J}^{k}} \sum_{\left\{n \mid(m, n) \in A^{j, k}\right\}} z_{m n}^{j, k} \leq 1, & \forall k \in \mathcal{K}, \forall j \in \mathcal{J}^{k}, \\
\sum_{\left\{n \mid(m, n) \in A^{j, k}\right\}} z_{m n}^{j k}=\sum_{\left\{n \mid(m, n) \in A^{j, k}\right\}} z_{n m}^{j k}, \forall k \in \mathcal{K}, \forall m \in\left(\mathcal{I}^{k} \cup \mathcal{J}^{k}\right), j \in \mathcal{J}^{k} \\
\sum_{\left\{n \mid(j, n) \in A^{j, k}\right\}} z_{j n}^{j k}=1, & \forall k \in \mathcal{K}, \forall j \in \mathcal{J}^{k}, \\
\sum_{\left\{m \mid(m, j) \in A^{j, k}\right\}} z_{m j}^{j k}=1, & \forall k \in \mathcal{K}, \forall j \in \mathcal{J}^{k}, \forall(m, n) \in \mathcal{A}^{j, k}, m \neq j, \\
z_{m n}^{j k}\left(T_{m}^{k}+\bar{t}_{m n}^{k}-T_{n}^{k}\right) \leq 0, & \forall k \in \mathcal{K}, \forall j \in \mathcal{J}^{k}, \forall(j, n) \in \mathcal{A}^{j, k}, \\
z_{j n}^{j k}\left(\bar{t}_{j n}^{k}-T_{n}^{k}\right) \leq 0, & \forall k \in \mathcal{K}, \forall m \in \mathcal{I}^{k}, \\
e_{m} \leq T_{m}^{k} \leq l_{m}, & \forall k \in \mathcal{K}, \forall j \in \mathcal{J}^{k}, \\
\sum_{(m, n) \in \mathcal{A}^{j, k}} \delta_{m n} z_{m n}^{j k} \leq D^{k}, & \forall k \in \mathcal{K}, \forall j \in \mathcal{J}^{k} . \\
\sum_{(m, n) \in \mathcal{A}^{j, k}} \Delta_{n}^{k} z_{m n}^{j k} \leq C^{k}, &
\end{array}
$$


Set $\mathcal{A}^{j, k}$ is introduced to ensure that the decision variable $z_{m n}^{j k}$ is defined properly for our problem. With that definition, the vehicles can only leave from and return to their own facilities. Also, they can only serve emergency points falling under the coverage area (defined by the distance ' $\mathrm{C}$ ') of their facilities. The objective function (10) is defined similar to (1) that it considers the priorities of each emergency. Relatedly, constraint (11) is relaxed from an equality to an inequality, in order to let emergency vehicles not serve lower priority emergencies and serve the higher priority ones when the demands are simultaneous. In the absolute priority case, VRPTW-P formulation ensures that emergency vehicles serve the higher priority demands even this might result in not serving lower priority demands independent of the number of lower priority demands. (11) imposes that every emergency point can be assigned to at most one single route according to the type of emergency vehicle demanded at that point. (12), (13), (14) describe the flow for the type-k vehicle from facility $j$. (15), (16), and (17) ensure that vehicle routes stay feasible with the given emergency time windows at each emergency point. (15) can be transformed into the following linear form:

$$
\left(T_{m}^{k}+\bar{t}_{m n}^{k}-T_{n}^{k}\right) \leq\left(1-z_{m n}^{j k}\right) M, \quad \forall k \in \mathcal{K}, \forall j \in \mathcal{J}^{k}, \forall(m, n) \in \mathcal{A}^{j, k}, m \neq j
$$

where $\mathrm{M}$ is a very large number. The same transformation could be used for (16) as well to keep the VRPTW-P as a linear integer problem. (18) guarantees feasibility for the range capacity, and (19) guarantees feasibility for the emergency relief material capacity of a vehicle.

\section{$3 \quad$ Emergency Response Planning}

Now that we have defined our location and routing models, MCLP-PB and VRPTW-P, we are ready to demonstrate how these two models could be utilized in preparation of real life ERPs. As mentioned earlier, for a problem like the ERP, where location and routing decisions will be interrelated, the facility location decisions should be assessed by how effectively the vehicles will respond to actual demands. In order to assess MCLP-PB's performance, we considered various types of emergency scenarios, and analyzed how well the allocated vehicles were able to serve the demands with VRPTW-P. In this assessment, we consider the performance of MCLP-P as a baseline to our model.

For our simulations we consider the 44-node network shown in Figure 1, where red colored nodes represent emergency points (EPs), and the remaining nodes possible locations for the emergency facilities. The network and EP locations are based on a typical metropolitan city, where high priority locations are clustered together in a central region, while low priority locations are sparsely distributed on the city fringes. For a concise argument, we assume that only two types of emergency vehicles, fire trucks and medical vehicles, are demanded at EPs. Each emergency type at each point has its priority, $p_{i}^{k}$, shown next to the EP, with higher values representing more important demands. In the network, there are a total of 14 priority level-1 (L1), 8 priority level-2 (L2), and 2 priority level-3 (L3) emergencies, with L3s having the highest priority. Again, for conciseness 
we let the number of distinct base coverage demand, $d_{i}^{k}$, equal to $p_{i}^{k}$, consistent with the fact that more important EPs requiring more distinct coverage.

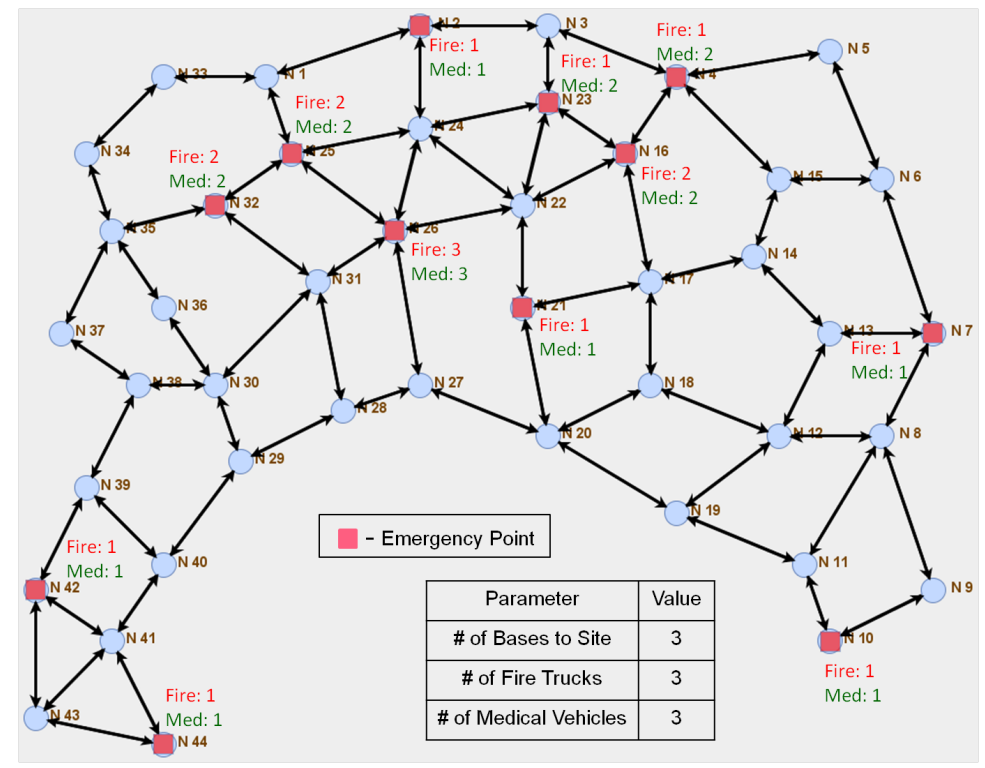

Fig. 1. 44-Node Simulation Network

\subsection{Siting of Emergency Facilities and Vehicles}

Both MCLP-P and MCLP-PB models are solved to optimality using a binary programming solver, and results are shown in Fig. 2 and Fig. 3, with facilities shown in yellow color. In the MCLP-P facility configuration Fig. 2, the facility sited at node-24, is able to cover all EPs at the upper- center of the network. The facilities sited at node-8 (right-bottom) and node-40 (left-bottom) cover the EPs at the fringes of the network. As a result, the facilities are more spread out, and all the EPs in the network are covered. In the facility configuration of the MCLP-PB model Fig. 3, the facilities are sited closer to the central high priority cluster, due to backup demands. As a result, some of the EPs located at the fringes of the network are not covered. For both configurations, each facility was fully operated, housing one fire truck and one medical vehicle each.

\subsection{Vehicle Routing Under Various Emergency Scenarios}

In this section, we consider various emergency scenarios to test our models. The scenarios vary in the arrival time of emergency demands, $A_{i}(i \in \mathcal{I})$, and the duration of service at EPs, $\lambda$, following a disaster. The variation in arrival times 


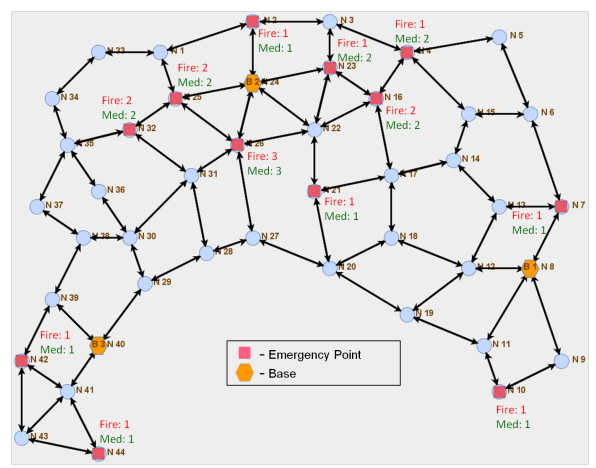

Fig. 2. MCLP-P Facility Configuration

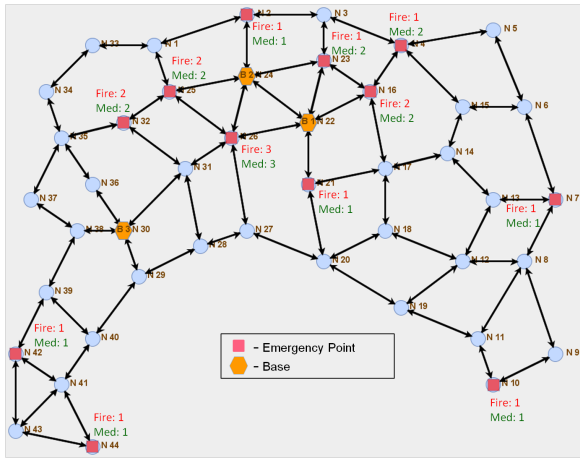

Fig. 3. MCLP-PB Facility Configuration

is related to the anticipation factor about an emergency, and is considered to see the performance of our models when some anticipation about an emergency's characteristics could be utilized. The variation in service duration is related to the severity of emergencies (more severe emergencies taking longer time to serve), and is considered to see the performance under emergencies of different severity.

Scenario 1 is modeled after considering a series of coordinated terrorist attacks, where an emergency response system would be suddenly overloaded by simultaneous emergency demands, without any possible earlier warning. Service duration, $\lambda$, is kept as a variable, and we assume $A_{i}=0, \forall i \in \mathcal{I}$, since there could be no warning (emergency demands would arrive instantaneously).

Scenario 2 is modeled after considering a spreading disaster, such as a flood with an estimated path of spread. In the simulation network, Fig. 1, we assume that the disaster spreads from the right nodes to left, with demands from EPs arriving in phases. The two rightmost EPs, at node- 7 and node-10, demand for vehicles at $A_{7}=0$ and $A_{10}=0$. As the disaster moves from right to left, the next two rightmost EPs, at node- 4 and node-16, demand vehicles at $A_{4}=\tau$, and $A_{16}=\tau$. Here $\tau$ denotes the time interval between phases, and is the variable defining the disaster spread speed. Subsequent demands from the EPs will arrive at $2 \tau, 3 \tau$, and so on. Based on this scenario, we consider two separate sub-scenarios Scenario 2a and 2b. In Scenario 2a, we assume that the demand arrival times for all EPs are known initially, so we can anticipate the arrival of the disaster at each EP. In Scenario 2b, we assume that the demand times are not known initially, and a demand only occurs when the location has been struck by a disaster. Hence, advance planning is not possible, and we can respond to these demands only when they occur.

In Scenario 3, each demand occurs in a random phase, and each demand will require service only once in the scenario. This scenario is modeled after a normal everyday situation, where demands for service occur independently of each other and the arrival of a demand is random in nature. As in the previous scenario, the time interval, $\tau$, determines the duration between arrival phases. For Scenarios $2 \mathrm{a}, 2 \mathrm{~b}$, and 3 , service duration $\lambda$ is assumed to be zero. 
As mentioned in the Introduction section, routing decisions for an ERP should be able to be updated during the actual emergencies with the arrival of new information (such as new emergency demands) in short decision times. For this reason, in this work we present a fast and highly accurate metaheuristic that solves the VRPTW-P. The details of the metaheuristic is given in Appendix 1, and it was was used to find the routes of all vehicles in all scenarios.

MCLP-P and MCLP-PB facility configurations was evaluated by the number of tasks successfully served, ordered by priority, by the vehicles dispatched from the facilities. Results of the simulated scenarios are displayed in Table 1.

Table 1. Comparison of MCLP-P and MCLP-PB

\begin{tabular}{|c|c|c|c|c|c|c|c|c|c|c|c|}
\hline \multirow{2}{*}{$\#$} & \multirow{2}{*}{ Scenario } & \multirow{2}{*}{ Parameters } & \multicolumn{3}{|c|}{ MCLP-P } & \multicolumn{3}{|c|}{ MCLP-PB } & \multicolumn{3}{|c|}{ MCLP-PB - MCLP-P } \\
\hline & & & L3 & $\mathrm{L} 2$ & L1 & L3 & L2 & L1 & L3 & L2 & L1 \\
\hline 1 & \multirow{4}{*}{1} & $\lambda=0.00 \mathrm{~h}$ & 2 & 4 & 7 & 2 & 8 & 5 & 0 & +4 & -2 \\
\hline 2 & & $\lambda=0.17 \mathrm{~h}$ & 2 & 3 & 5 & 2 & 7 & 2 & 0 & +4 & -3 \\
\hline 3 & & $\lambda=0.33 \mathrm{~h}$ & 2 & 1 & 4 & 2 & 5 & 0 & 0 & +4 & -4 \\
\hline 4 & & $\lambda=0.50 \mathrm{~h}$ & 2 & 0 & 2 & 2 & 3 & 0 & 0 & +3 & -2 \\
\hline 5 & \multirow{4}{*}{$2 \mathrm{a}$} & $\tau=0.05 \mathrm{~h}$ & 2 & 5 & 7 & 2 & 8 & 6 & 0 & +3 & -1 \\
\hline 6 & & $\tau=0.10 \mathrm{~h}$ & 2 & 5 & 6 & 2 & 8 & 6 & 0 & +3 & 0 \\
\hline 7 & & $\tau=0.15 \mathrm{~h}$ & 2 & 4 & 7 & 2 & 8 & 4 & 0 & +4 & -3 \\
\hline 8 & & $\tau=0.20 \mathrm{~h}$ & 2 & 4 & 3 & 2 & 7 & 4 & 0 & +3 & +1 \\
\hline 9 & \multirow{4}{*}{$2 \mathrm{~b}$} & $\tau=0.05 \mathrm{~h}$ & 2 & 3 & 7 & 2 & 8 & 5 & 0 & +5 & -2 \\
\hline 10 & & $\tau=0.10 \mathrm{~h}$ & 2 & 5 & 6 & 2 & 5 & 5 & 0 & 0 & +1 \\
\hline 11 & & $\tau=0.15 \mathrm{~h}$ & 1 & 3 & 6 & 2 & 4 & 6 & +1 & +1 & 0 \\
\hline 12 & & $\tau=0.20 \mathrm{~h}$ & 0 & 4 & 6 & 2 & 4 & 4 & +2 & 0 & -2 \\
\hline 13 & \multirow{4}{*}{3} & $\tau=0.05 \mathrm{~h}$ & 2 & 2 & 8 & 2 & 8 & 6 & 0 & +6 & -2 \\
\hline 14 & & $\tau=0.10 \mathrm{~h}$ & 2 & 5 & 7 & 2 & 5 & 7 & 0 & 0 & 0 \\
\hline 15 & & $\tau=0.15 \mathrm{~h}$ & 1 & 3 & 7 & 2 & 3 & 7 & 1 & 0 & 0 \\
\hline 16 & & $\tau=0.20 \mathrm{~h}$ & 2 & 2 & 8 & 2 & 3 & 7 & 0 & +1 & -1 \\
\hline
\end{tabular}

For Scenario 1, 2a and 3, the MCLP-P facility configuration results in vehicles serving more L1 EPs, while the MCLP-PB facility configuration results in vehicles serving more L2 EPs. It should be noted that for Scenario 1, even when the service duration $\lambda$ is 0.5 hours, which is in fact half of the time limit assumed for facility coverage in these simulations, the vehicles in the MCLP-PB facility configuration are still able to serve $3 \mathrm{~L} 2 \mathrm{EPs}$, whereas none of the vehicles in the MCLP-P facility configuration are able to serve any L2 EP (Table 1, row 4).

In Scenario 2b, it is interesting to observe that when the time interval between phases increases, even the most important L3 EPs are dropped in the MCLP$\mathrm{P}$ facility configuration (Row 11 and 12 of Table 1). On the other hand, with the MCLP-PB facility configuration, all the L3 EPs are always served. When the time interval is large, an emergency vehicle is more likely to be en route to a lower priority EP when a new demand arrives from a higher priority EP. If vehicles are too far away from the higher priority EPs, they will be unable to service those, resulting in dropped demands from the higher priority EPs. In 
contrast, with the MCLP-PB facility configuration, the backup facilities (and vehicles housed in them) are able to provide service to the higher priority EPs' demands.

\subsection{Integrated Analysis}

In the previous section, we compared the effectiveness of emergency vehicles' response to simulated emergencies from the facility configurations found by MCLP$\mathrm{P}$ and MCLP-PB models. Notice that the facility locations did not change after the routing analysis was completed, so the overall planning was in fact carried out in an open loop manner. As mentioned in the Introduction section, this type of independent FLP-VRP solution process could result in an overall sub-optimal solution. For this reason, we designed an extension to this process to include a feedback loop to find better facility configurations. For this analysis, a new method to generate alternate facility configurations based on the solution of the routing algorithm is developed. In this method, we shift the emergency facilities to other possible locations that could improve vehicles' responses to emergencies. This method is described in greater detail Appendix 2.

We conducted additional simulations with Scenario 1 and 2 a to observe the effectiveness of the proposed closed-loop planning method. The results of the alternate facility configuration simulations are shown in Table 2.

Table 2. Comparison with Facility Shifting

\begin{tabular}{|c|c|c|c|c|c|c|c|c|c|c|c|c|c|c|}
\hline \multirow{2}{*}{ \# } & \multirow{2}{*}{ Scenario } & \multirow[b]{2}{*}{ Parameters } & \multicolumn{3}{|c|}{ MCLP-P } & \multicolumn{3}{|c|}{ MCLP-P shift } & \multicolumn{6}{|c|}{\begin{tabular}{|l|l|} 
MCLP-PB & MCLP-PB shift
\end{tabular}} \\
\hline & & & L3 & $\mathrm{L} 2$ & L1 & L3 & $\mathrm{L} 2$ & L1 & & 2 & L1 & L3 & L2 & L1 \\
\hline 1 & \multirow{4}{*}{1} & $\lambda=0.00 \mathrm{~h}$ & 2 & 4 & 7 & 2 & 4 & 7 & 2 & 8 & 5 & 2 & 8 & 5 \\
\hline 2 & & $\lambda=0.17 \mathrm{~h}$ & 2 & 3 & 5 & 2 & 3 & 6 & 2 & 7 & 2 & 2 & 8 & 2 \\
\hline 3 & & $\lambda=0.33 \mathrm{~h}$ & 2 & 1 & 4 & 2 & 1 & 5 & 2 & 5 & 0 & 2 & 6 & 1 \\
\hline 4 & & $\lambda=0.50 \mathrm{~h}$ & 2 & 0 & 2 & 2 & 0 & 4 & 2 & 3 & 0 & 2 & 4 & 0 \\
\hline 5 & \multirow{4}{*}{$2 \mathrm{a}$} & $\tau=0.05 \mathrm{~h}$ & 2 & 5 & 7 & 2 & 5 & 7 & 2 & 8 & 6 & 2 & 8 & 6 \\
\hline 6 & & $\tau=0.10 \mathrm{~h}$ & 2 & 5 & 6 & 2 & 5 & 6 & 2 & 8 & 6 & 2 & 8 & 6 \\
\hline 7 & & $\tau=0.15 \mathrm{~h}$ & 2 & 4 & 7 & 2 & 4 & 7 & 2 & 8 & 4 & 2 & 8 & 4 \\
\hline 8 & & $\tau=0.20 \mathrm{~h}$ & 2 & 4 & 3 & 2 & 4 & 6 & 2 & 7 & 4 & 2 & 8 & 4 \\
\hline
\end{tabular}

As can be noticed from the results, when facility shifting is allowed, there is a small improvement in the number of EPs served by the vehicles for all scenarios and for both base configurations. These results show the potential improvements to emergency plans by utilizing an integrated location-routing analysis particularly for emergencies whose characteristics could be estimated beforehand, such as a flood with an estimated path.

\section{Conclusions}

In this paper, for emergency response preparation, we have formulated new location, MCLP-PB, and routing, VRPTW-P, models with priority considerations. 
MCLP-PB, also utilizes the extra backup coverage concept to introduce robustness to the facility network. A fast and highly accurate metaheuristic, that could be utilized in real life emergencies, is developed for VRPTW-P. We tested our formulations on a 44-node network that is influenced from a metropolitan city. The MCLP-PB model generated facility configurations that are better in achieving the goal of servicing higher priority EPs, as compared to the baseline MCLP$\mathrm{P}$ model. While the MCLP-PB model does neglect the coverage of some lower priority EPs, in crisis management where resources could be scarce and high priority EPs needs to be addressed first, the MCLP-PB model is better able to achieve this goal. We have also proposed an integrated feedback type algorithm involving facility shifting. The algorithm for this integrated model performed better than the algorithms for both the MCLP-P and MCLP-PB models under various scenarios. While this study is developed with the ERP problem in mind, our approach would also be useful for other location problems where priority is a major concern, and the location and routing decisions are interrelated.

\section{References}

1. L. Brotcorne, G. Laporte, and F. Semet, "Ambulance location and relocation models," European J. of Operational Research, vol. 147, no. 3, pp. 451 - 463, 2003.

2. B. Eksioglu, A. V. Vural, and A. Reisman, "The vehicle routing problem: A taxonomic review," Computers \& Industrial Engineering, vol. 57, no. 4, 2009.

3. "Principles of emergency management supplement." http://training.fema.gov/ EMIWeb/edu/emprinciples.asp, September 2008.

4. F. Silva and D. Serra, "Locating emergency services with different priorities: the priority queuing covering location problem," J. Op. Res. Soc., vol. 59, no. 9, 2008.

5. K. Hogan and C. ReVelle, "Concepts and applications of backup coverage," Manage. Sci., vol. 32, pp. 1434-1444, November 1986.

6. J. R. OHanley and R. L. Church, "Designing robust coverage networks to hedge against worst-case facility losses," European J. of Op. Res., vol. 209, no. 1, 2011.

7. A. Klose and A. Drexl, "Facility location models for distribution system design," European J. of Operational Research, vol. 162, no. 1, pp. 4 - 29, 2005.

8. J. Desrosiers, Y. Dumas, M. M. Solomon, and F. Soumis, "Chapter 2 time constrained routing and scheduling," in Network Routing, vol. 8 of Handbooks in Op. Res. ES Man. Science, pp. 35 - 139, Elsevier, 1995.

9. J. Cordeau, G. Laporte, and a. Mercier, "A unified tabu search heuristic for vehicle routing problems with time windows," J. Op. Res. Soc., vol. 52, Aug. 2001.

10. M. Solomon, "Algorithms for the vehicle routing and scheduling problems with time window constraints," Operations research, vol. 35, no. 2, pp. 254-265, 1987.

\section{Appendix 1: Vehicle Routing Problem Heuristics}

For emergency vehicle routing, we use a tabu search algorithm adapted from the unified tabu search (UTS) algorithm in [9]. In the original UTS algorithm, the solution produced by the initial construction phase is not guaranteed to be feasible. We modified the initial construction phase of the UTS algorithm to use 
the I1 insertion heuristic from [10], so that the algorithm can return a feasible solution at any time.

In the original UTS algorithm, all EPs have equal priority. We modified the algorithm to consider absolute priorities. During the search, we compare solutions as follows. Suppose the EP priorities from lowest to highest are $1,2, \cdots, p^{*}$. The most important criterion is to maximize the number of priority $p^{*}$ EPs assigned to a solution. The next most important criterion is to maximize the number of priority $\left(p^{*}-1\right)$ EPs assigned to a solution, and this proceeds until the priority 1 EPs have been considered. Finally, the least important criterion is to minimize the total distance of all the routes in a solution, for a total of $p^{*}+1$ criteria. Finally, we adapted the algorithm to be able to perform replanning whenever the situation changes.

Our metaheuristic algorithm performed well on the well-studied Solomon benchmark set of problems [10]. This benchmark set contains over 150 VRPTW instances with differing characteristics in terms of size, location distribution and time window duration, and has mostly been solved optimally, with minimizing total distance as the main criterion. For every instance, our algorithm produces a solution within $10 \%$ of optimality, meaning that the total distance of the solution is within $110 \%$ of the optimal total distance. For smaller problem instances containing 25 locations, our algorithm takes only a few seconds. For larger problem instance containing 100 locations, our algorithm takes less than 5 minutes. It is important to note that this benchmark set only contains static problems, and does not have locations of different priorities. However, our algorithm's strong performance on this data set together with its ability to perform replanning makes it suitable for our purposes.

\section{Appendix 2: Finding Alternate Facility Configurations}

Using the result from the routing algorithm, better alternate facility configurations can be generated. A tabu list of facility configurations is used to widen the exploration of the search space. The facility to relocate is the one covering the dropped highest priority EP. The neighboring nodes to the facility and the nodes on the shortest path between the facility and the dropped EP are put into a list of possible locations to shift to.

In the list, nodes with a facility are dropped from the list. If the facility is relocated to a node that results in a tabu configuration, the node is also removed from the list of possible locations. From the filtered list, the one possible location to consider for relocation is selected by finding the location that can cover the most priority weighted EPs. After obtaining the possible location, we shift the facility if the dropped EP has a priority that is higher or equal to all the EPs that are served by the facility. Otherwise, we will only shift the facility if all the higher level EPs that is served by the facility can still be covered. Once we decide to shift the facility, the current facility configuration is added to the tabu list and the alternate facility configuration is now used as the input into the routing algorithm. 